\title{
REFERENCES
}

1. S. Bochner and A. E. Taylor, Linear functionals on certain spaces of abstractlyvalued functions, Ann. of Math. (2) 39 (1938), 913-944.

2. Avner Friedman, Convergence of solutions of parabolic equations to a steady state, J. Math. Mech. 8 (1959), 57-76.

3. O. A. Ladyženskaja and N. N. Ural'ceva, $A$ boundary-value problem for linear and quasi-linear parabolic equations. I, Izv. Akad. Nauk SSSR Ser. Mat. 26 (1962), 5-52. (Russian)

4. W. Littman, G. Stampacchia and H. F. Weinberger, Regular points for elliptic equations with discontinuous coeficients, University of Minnesota Report, Minneapolis, Minn., December 1962.

5. J. Nash, Continuity of solutions of parabolic and elliptic equations, Amer. J. Math. 80 (1958), 931-954.

6. W. Pogorzelski, Etude d'une fonction de Green et du probleme aux limites pour l'équation parabolique normale, Ann. Polon. Math. 4 (1958), 288-307.

7. G. Stampacchia, Régularisation des solutions de problèmes aux limites elliptiques a données discontinues, Internat. Sympos. on Linear Spaces, Pergamon, Jerusalem, 1960.

UNIVERSITY OF MinNESOTA

\section{ON APPROXIMATE SOLUTIONS TO THE CONVOLUTION EQUATION ON THE HALF-LINE}

\author{
BY T. K. BOEHME
}

Communicated by R. C. Buck, July 24, 1963

1. We will call a complex-valued function on the half-line $t>0$ locally integrable if it is integrable on each interval $[0, T], T>0$. Let $\&$ be the ring of locally integrable functions (functions which are equal up to a set of measure zero will be identified with each other) with the usual pointwise addition, and with convolution for the product operation. Thus $k x=r$ if and only if $\int_{0}^{t} k(t-u) x(u) d u=r(t)$ for almost every $t>0$. Give $\&$ the topology defined by the seminorms $\|x\|_{T}=\int_{0}^{T}|x|(u) d u, T>0$. Thus a sequence $x_{n}, n=1,2, \cdots$ in $\&$ converges to 0 in $\&$ if and only if $x_{n} \rightarrow 0$ in $L[0, T]$ for each $T>0$ as $n \rightarrow \infty$. The equation $k x=r$ is an important integral equation; however, solutions and the existence of solutions are in general difficult to obtain. M. I. Fenyö and C. Foias [1] ${ }^{1}$ have shown that if $k$ and $r$ are in $\mathscr{L}$ and if $k$ vanishes on no neighborhood of the origin (i.e. $\|k\|_{T}>0$ for each $T>0$ ) there is always an approximate solution to the equation

1 The author thanks the referee for calling this article to his attention. 
$k x=r$ in the sense that if $T>0$ and $\epsilon>0$ are given there is an $x$ in $L[0, T]$ such that $\|r-k x\|_{T}<\epsilon$. We shall give a new proof of this result which enables one to see how such approximate solutions can be constructed, when $k$ is a real function, in terms of the characteristic functions of a completely continuous self-adjoint operator on a Hilbert space.

2. Each element $k$ in $\mathscr{L}$ defines a continuous linear transformation of $\mathcal{L}$ into $\mathcal{L}$, and of $L^{p}[0, T]$ into $L^{p}[0, T]$ (for each $T>0,1 \leqq p \leqq \infty$ ) by the formula $K(x)=k x$. Let $\bar{K}$ be the transformation defined by the complex conjugate $\bar{k}$ of $k$, and denote by $S$ the transformation of $L^{p}[0, T]$ into $L^{p}[0, T]$ which is defined by $S(x)(t)=x(T-t)$ for all $t$ in $[0, T]$. If $K$ is considered as an operator on the space $L[0, T]$ it is easy to verify that the adjoint transformation of $K$ is $K^{*}=S \bar{K} S$. By a well-known theorem of Titchmarsh if $k$ does not vanish on a neighborhood of the origin then $K(x)=0$ in $L[0, T]$ if and only if $x$ is the zero element of $L[0, T]$; thus the null space of $K^{*}=S \bar{K} S$ consists of the zero element alone and the range of $K$ is dense in $L[0, T]$.

We will call a sequence $x_{n}, n=1,2, \cdots$, in $\&$ an approximate solution to the equation $k x=r$ if $k x_{n} \rightarrow r$ in $\mathscr{L}$ as $n \rightarrow \infty$. We have proved the following theorem.

TheOREM (FoIAş). If $k$ in $\&$ vanishes on no neighborhood of the origin and $r$ is in $\&$ there is an approximate solution in $\&$ to the equation $k x=r$.

3. Henceforth we shall consider $k$ to be real and to vanish on no neighborhood of the origin. ${ }^{2}$ In order to construct approximate solutions we consider $K$ and $S$ as operators on the Hilbert space $L^{2}[0, T]$. The operator $K S$ is self-adjoint since $(K S)^{*}=S^{*} K^{*}=S(S K S)=K S$. Moreover, if $B$ is any bounded set in $L^{2}[0, T]$ the set $\{k x \mid x$ in $B\}$ is bounded and is equicontinuous in norm; thus, it has compact closure. It follows that $K$ is completely continuous, and consequently $K S$ is a completely continuous self-adjoint operator on the Hilbert space $L^{2}[0, T]$. Let $\lambda_{n}, n=1,2, \cdots$, be the characteristic values of $K S$ and let $\phi_{n}, n=1,2, \cdots$, be the corresponding orthonormal characteristic functions. Since the null space of $K S$ consists of the zero element alone, the characteristic functions $\phi_{n}$ form a complete orthonormal system for $L^{2}[0, T]$. The equation $k x=f$ with $k$ in $L[0, T]$ and $f$ in $L^{2}[0, T]$ has a solution in $L^{2}[0, T]$ if and only if

${ }^{2}$ If $k$ is not real, i.e. $K S$ is not self-adjoint, essentially these same methods can be used. See F. Smithies, Integral equations, Cambridge Tracts in Mathematics and Mathematical Physics, No. 49, Chapter VIII. 
$\sum_{0}^{\infty}\left|\left(f, \phi_{n}\right) / \lambda_{n}\right|^{2}<\infty$, and if this quantity is finite that solution is given by

$$
x=\sum_{0}^{\infty} \frac{\left(f, \phi_{n}\right)}{\lambda_{n}} S\left(\phi_{n}\right) .
$$

Even if there is no solution in $L^{2}[0, T]$ the functions

$$
x_{f, N}=\sum_{0}^{N} \frac{\left(f, \phi_{n}\right)}{\lambda_{n}} S\left(\phi_{n}\right) \quad N=1,2, \cdots
$$

are such that $K\left(x_{N}\right)=\sum_{0}^{N}\left(f, \phi_{n}\right) \phi_{n} \rightarrow f$ in $L^{2}[0, T]$ and a fortiori in $L[0, T]$ as $N \rightarrow \infty$. We can now construct an approximate solution to the equation $k x=r$. For each positive integer $i$ pick $f_{i}$ in $L^{2}[0, i]$ such that $\left\|r-f_{i}\right\|_{i}<1 / i$, and for each $i$ take $N_{i}$ such that $\left\|f_{i}-x_{f_{i}, N_{i}}\right\|_{i}<1 / i$. The functions $x_{i}$ which are such that $x_{i}(t)=x_{f_{i}, N_{i}}(t)$ on $[0, i]$ and $x_{i}(t)=0$ for $t>i$ constitute an approximate solution to the equation $k x=r$.

4. For $0<\alpha<\infty$ let $\mathfrak{L}_{\alpha}=\left\{x \mid x \in \mathcal{L}\right.$ and $\left.\|x\|_{\alpha}=0\right\}$.

Corollary 1. $A$ is a closed proper ideal in $\mathfrak{L}$ if and only if $A=\mathfrak{L}_{\alpha}$ for some $\alpha$.

Proof. Clearly each $\mathscr{L}_{\alpha}$ is a closed proper ideal. If $A$ is a closed proper ideal in $\&$ let $\bar{\beta}=\inf \left\{\beta \mid \beta>0, \exists x \in A,\|x\|_{\beta}>0\right\} . \bar{\beta}$ is a nonnegative number, and by the above theorem $A$, being closed, contains $\mathfrak{L}_{\beta}$ for each $\beta>\bar{\beta}$. Since $A$ is closed and not equal to $\mathscr{L}, \bar{\beta}$ is not zero and $A=£ \bar{\beta}$.

In particular there are no proper maximal ideals in $\&$ or in any of the Banach algebras $L[0, T], T>0$. This yields a theorem of RyllNardzewski [2]:

Corollary 2. For $f \in \mathfrak{L}, f^{n} \rightarrow 0$ in $\mathcal{L}$ as $n \rightarrow \infty$.

PRoof. Since $L[0, T]$ has no maximal ideals the spectral radius of $f \in L[0, T]$ equals zero, and thus $\left\|f^{n}\right\|_{T \rightarrow 0}$ as $n \rightarrow \infty$ for each $T>0$.

\section{REFERENCES}

1. C. Foiaş, Approximation des opérateurs de J. Mikusinski par des fonctions contiues, Studia Math. 21 (1961), 73-74.

2. Cz. Ryll-Nardzewski, Sur les séries de puissances dans le calcul opératoire, Studia Math. 13 (1953), 41-47.

UNIVERSITY OF WASHINGTON AND The Boeing Company 